OPEN ACCESS

Edited by:

Antonio Di Sabatino,

University of Pavia, Italy

Reviewed by:

Silvia Melgar,

University College Cork, Ireland

Ludvig M. Sollid,

University of Oslo, Norway

*Correspondence:

Ruggiero Francavilla

ruggiero.francavilla@uniba.it

Specialty section:

This article was submitted to Nutritional Immunology,

a section of the journal

Frontiers in Immunology

Received: 30 May 2020 Accepted: 29 September 2020 Published: 03 November 2020

Citation:

Cristofori F, Francavilla $R$

Capobianco D, Dargenio VN, Filardo S and Mastromarino $P(2020)$

Bacterial-Based Strategies to

Hydrolyze Gluten Peptides and

Protect Intestinal Mucosa.

Front. Immunol. 11:567801. doi: 10.3389/fimmu.2020.567801

\section{Bacterial-Based Strategies to Hydrolyze Gluten Peptides and Protect Intestinal Mucosa}

\author{
Fernanda Cristofori ${ }^{1}$, Ruggiero Francavilla ${ }^{1 *}$, Daniela Capobianco ${ }^{2}$, \\ Vanessa Nadia Dargenio ${ }^{1}$, Simone Filardo ${ }^{2}$ and Paola Mastromarino ${ }^{2}$ \\ 1 Interdisciplinary Department of Medicine-Pediatric Section, Università di Bari Aldo Moro, Bari, Italy, ${ }^{2}$ Department of Public \\ Health and Infectious Disease, Università La Sapienza di Roma, Rome, Italy
}

Gluten is a mixture of proteins highly resistant to hydrolysis, resulting in the emergence of toxic peptides responsible for gluten-related disorders. Currently, a gluten-free diet (GFD) is the unique proven therapy for celiac disease (CD). Several research groups and pharmaceutical companies are developing new nondietetic therapeutic strategies for CD. Probiotics are viable microorganisms thought to have a healthy effect on the host. The proteolytic mechanism of lactic acid bacteria comprises an extracellular serine protease, di- and oligopeptide-specific transport systems, and several intracellular peptidases that might affect gluten degradation. Therefore, probiotic supplementation is an attractive therapy because of its possible anti-inflammatory and immunomodulatory properties. Several studies have been performed to assess the effectiveness of various specific probiotic strains, showing positive effects on immune-modulation (inhibition of proinflammatory cytokine TNF- $\alpha$ ) restoring gut microbiota and decrease of immunogenic peptides. The present review aims to summarize the current knowledge on the ability of probiotic strain (single or mixtures) to digest gliadin peptides in vitro and to modulate the inflammatory response in the gut.

Keywords: celiac disease, microbiota, probiotics, gluten hydrolysis, gluten digestion

\section{INTRODUCTION}

Celiac disease (CD) is an autoimmune enteropathy that occurs in genetically predisposed individuals who develop an immune response after gluten ingestion (1). The prevalence of this condition, estimated at around $1 \%$, is increasing worldwide. The gold standard in CD treatment relies on a gluten-free diet (GFD) that needs to be strict and lifelong and should be directed by a specialist nutritionist (2); although novel nondietetic treatments are under study, GFD is the only available therapy for $\mathrm{CD}$ at present.

$\mathrm{CD}$ is a polygenic disease with a strict gene environmental interaction, and recently, the role of gut microbiota (GM) has become of primary interest. Available literature supports the theory that modification in GM is related to many chronic inflammatory diseases, including obesity, diabetes, inflammatory bowel disease, and CD (3). The role of the GM in CD is supported by evidence that germ-free mice develop a gluten-related pathology whose severity depends on the type of microbial 
colonization in their guts (4), hence the idea that GM modulation with the use of probiotics can be used for therapeutic purposes in the course of CD.

The information describing the gut microbiota in CD patients has been derived from both fecal samples and duodenal mucosa biopsies generally in children with CD. Various authors have found contrasts in the microbial composition of biological samples when comparing active $\mathrm{CD}$ to patients adherent to the GFD or healthy controls. The following findings (demonstrated by more than two independent evidences) supports the association between CD and GM: 1) increased Bacteroidetes in biopsies and stools of active and inactive CD, 2) decreased fecal Bifidobacterium spp. in active and inactive CD, 3) increased Proteobacteria in biopsies and stools of active CD, and 4) increased Staphylococcus in biopsies and stools of active CD (5).

"Probiotics are 'live microorganisms' that confer, if administered in adequate amounts, benefit(s) to the host health" (6). Probiotic mechanisms of action include modulation of GM, suppression of potentially harmful microorganisms (producing antimicrobial or other factors, thus suppressing growth or competing for mucosal receptors of pathogens), regulation of the immune system, and mucosal barrier strengthening.

Therefore, several studies look at the possible use of probiotics in $\mathrm{CD}$ as complementary treatment to modulate the microbiome or detoxify gluten (7).

\section{PROBIOTICS AND WHEAT DEGLUTINATION}

Gliadin and glutenin (gluten immunogenic peptides) are the major antigens leading to intestinal damage in CD. Gliadin and glutenin are rich in proline and glutamine residues and are able to resist gastric, pancreatic, and intestinal proteolysis. Some of these immunogenic peptides can induce the inflammatory process associated with $\mathrm{CD}(8,9)$.

The human GM includes bacterial species able to degrade gluten peptides, affecting their toxicity (10-12). However, the microbiota may either be protective against or contribute to the development of CD through the generation of harmful immunogenic peptides $(13,14)$.

Indeed, strains of Bacteroides fragilis, isolated from the GM of $\mathrm{CD}$ patients, display gliadin-hydrolyzing activity, some of them giving rise to immunogenic peptides and, hence, inducing inflammatory cytokine production by intestinal epithelial cells (13). Moreover, Pseudomonas aeruginosa isolates, recovered from the duodenum of $\mathrm{CD}$ patients, produced, via their elastase activity, several peptides that elicited the activation of gluten-specific T-cells in these patients. By contrast, Lactobacillus spp, isolated from healthy individuals, degraded $P$. aeruginosamodified peptides and reduced their immunogenicity (14).

Lactobacilli and bifidobacteria are considered essential intestinal microbial genera that have beneficial effects on human health and are widely used in the formulation of probiotic products. Therapy with probiotics, containing bacteria capable of degrading gluten, is a possible new strategy for the complementary treatment of $\mathrm{CD}$ patients. Indeed, a compelling approach could consist of the in situ detoxification of gluten by resident intestinal bacteria through their metabolic activity.

Several publications have reported the results of in vitro and in vivo studies on lactobacilli and bifidobacteria strains capable of hydrolyzing gluten proteins (Table 1, Figure 1) reducing their toxicity and/or inflammatory effect. Most of the studies are carried out on different strains and species of lactobacilli, identified as the principal gluten-metabolizing bacteria in the gut $(10,11)$. Indeed, it is suggested that lactobacilli possessed a competitive advantage on other microorganisms due to the property to produce nitrogen from gluten (10): Extracellular and cell wall-associated proteases cleave proteins into oligopeptides, carried across the cell membrane and then hydrolyzed by intracellular peptidases (21).

A mixture of Lactobacillus alimentarius 15M, Lactobacillus brevis 14G, Lactobacillus sanfranciscensis 7A, and Lactobacillus hilgardii 51B, chosen for their distinct hydrolysis profiles toward wheat gliadins, were capable of fully hydrolyzing the fragment 62-75 of A-gliadin and the 33-mer peptide (15). The probiotic mixture showed iminopeptidase, dipeptidyl-peptidase, prolyl endopeptidase, prolidase, prolinase, and aminopeptidase $\mathrm{P}$ enzymatic activities. The effectiveness of lactobacilli on wheat deglutination was verified by in vivo acute challenge in $C D$ patients on a GDF. As a result, these patients did not display any gut permeability impairment after eating bread produced with lactobacilli-fermented wheat flour.

Subsequent studies have confirmed that the simultaneous presence of proteases with different specificities is necessary to obtain complete hydrolysis of the toxic peptides derived from gliadin. The combined activity of general aminopeptidase type $\mathrm{N}$ (PepN; EC 3.4.11.11), endopeptidase (PepO; EC 3.4.23), and prolyl endopeptidyl peptidase (PEP; EC 3.4.21.26), obtained from selected sourdough lactobacilli, induced the hydrolysis of the CD immunogenic 33-mer peptide into five small peptides (22). Overall, five peptidases were necessary to fully metabolize the 33-mer and other synthetic immunogenic peptides (22). Previously, the same group demonstrated that a probiotic preparation, containing Streptococcus thermophilus, Lactobacillus plantarum, Lactobacillus acidophilus, Lactobacillus casei, Lactobacillus delbrueckii subsp. bulgaricus, Bifidobacterium breve, Bifidobacterium infantis, and Bifidobacterium longum, reduced wheat flour toxicity during sourdough fermentation for an extended period (16). However, the ability of probiotic microorganisms to hydrolyze gliadin peptides was missing when the individual strains were assayed. These studies suggest that no individual bacterial strain have the whole spectrum of peptidases required to degrade the different peptides contributing to $\mathrm{CD}$.

On the other hand, the healthy GM is a very complicated ecosystem characterized by several bacterial species that can be involved in the degradation of gliadin peptides through enzymes that are not produced by humans. Therefore, the different kind of 
TABLE 1 | In vitro and in vivo studies on lactobacilli and bifidobacteria strains capable of hydrolyzing gluten proteins reducing their toxicity and/or inflammatory effect.

Mixture of Lactobacillus alimentarius, L. Iminopeptidase, dipeptidyl-peptidase, Complete hydrolysis of $\alpha 2$-gliadin-derived brevis, L. sanfranciscensis, L. hilgardii

Mixture of Streptococcus thermophilus, Lactobacillus plantarum, L. acidophilus, L. casei, L. delbrueckii subsp.

bulgaricus, Bifidobacterium breve, $B$. infantis, B. longum

Mixture of $L$. casei, L. delbrueckil

subsp. bulgaricus, L. paracasei LPC01

and BGP2, LP35, LP40, LP47, and SP1

Mixture of Lactobacillus paracasei, $L$. plantarum, Bifidobacterium animalis

subsp. lactis, B. breve Bbr8, B. breve

BL10

Bifidobacterium animalis, B. longum, $B$. -

bifidum

Bifidobacterium bifidum, B. longum, B. breve, $B$. animalis individual species

and as mixture

prolylendopeptidase, prolidase, prolinase, epitopes 62-75 and 33-mer aminopeptidase $\mathrm{P}$

Proline iminopeptidase, aminopeptidase Complete hydrolysis of $\alpha 2$-gliadin-derived

$\mathrm{N}$ and $\mathrm{A}$, dipeptidase, prolinase, prolidase, dipeptidyl peptidase,

tripeptidase, prolylendopeptidase

endopeptidase endopeptidyl peptidase, tripeptidase

prolidase, prolinase, dipeptidase
Aminopeptidase N, iminopeptidase, prolyl Complete hydrolysis of $\alpha 9$-gliadin peptide 57-
68, 33-mer, A-gliadin peptide 62-75, $\gamma$-gliadin peptide $62-75$

Absence of immunogenic peptides after hydrolysis of the wheat bread gluten

Hydrolysis of gliadin peptides generated by

digestive proteases into smaller fragments.

Hydrolysis of 33-mer

Different peptide patterns with lower molecular mass compared to those noninoculated with bacteria

Hydrolysis of gluten in different peptide patterns
No alteration of intestinal permeability after ingestion of bread produced with wheat flour fermented with lactobacilli in CD patients on a GDF Reduced alteration of gliadin-induced increase of intestinal cell cultures permeability

Reduction to basal level of IL-2, IL-10, and IFN- $\gamma$ increase induced by untreated wheat bread gluten in duodenal biopsy specimens from CD patients

Inhibition of the inflammatory state and disruption of Giorgi et al. (18) tight junctions induced by gliadin in intestinal

epithelial cells.

Reduction of the cytotoxic effect and inhibition of NF-kB activation and TNF- $\alpha$ production induced by gliadin in intestinal epithelial cells especially by $B$. longum.

Reduction of the cytotoxic effect and inhibition of

NF-kB activation and TNF- $\alpha$ production induced by et al. (20)

gluten in intestinal epithelial cells especially by $B$.

longum and the Bifidobacterium consortium
Di Cagno et al. (15)

De Angelis et al. (16)

Francavilla et al. (17) 


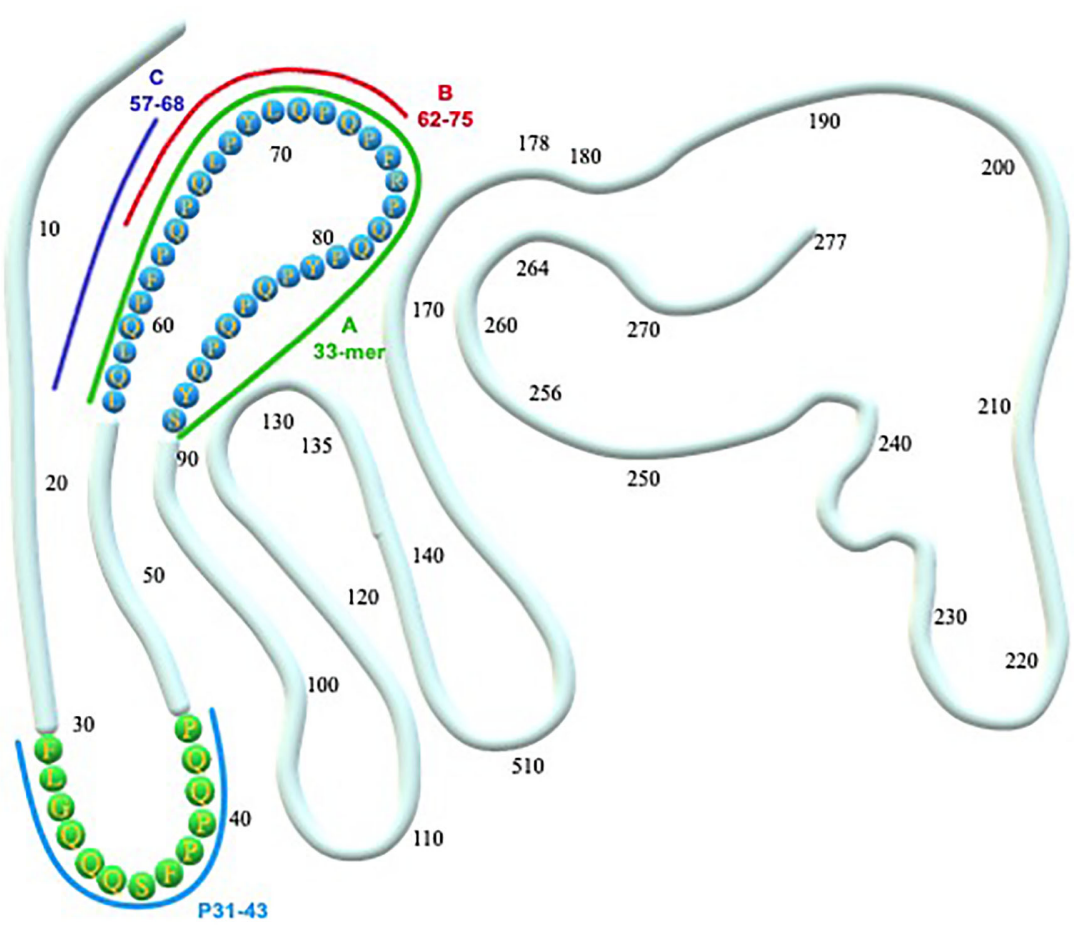

FIGURE 1 | $\alpha$-gliadin structure and toxic epitopes (33-mer and p31-43). A, B, C represent the peptides hydrolyzed by a different mixture of probiotic strains. (A) Hydrolyzed by a mixture of L. paracasei, L. plantarum, Bifidobacterium animalis subsp. lactis, B. breve Bbr8, B. breve BL10 (18). (A, B) Hydrolyzed by a mixture of L. alimentarius, L. brevis, L. sanfranciscensis, L. hilgardii (15); mixture of Streptococcus thermophilus, L. plantarum, L. acidophilus, L. casei, L. delbrueckii subsp. bulgaricus, Bifidobacterium breve, B. infantis, B. longum (16). (A-C) Hydrolyzed by a mixture of L. casei, L. delbrueckii subsp. bulgaricus, L. paracasei LPC01 and BGP2, L. plantarum BGP12, LP27, LP35, LP40, LP47, and SP1 (17).

proteases found in the gut, each with its specificity, could be responsible for the complete hydrolysis of toxic gliadin peptides.

Recently, several lactobacilli strains (L. casei BGP93; L. delbrueckii subsp. bulgaricus SP5; L. paracasei LPC01, BGP1, and BGP2; L. plantarum BG112, BGP12, LP27, LP33, LP35, LP36, LP39, LP40, LP42, LP47, and LP32; L. rhamnosus SP1; and L. reuteri DSM17938) have been evaluated for the ability to hydrolyze immunogenic gluten peptides. A highly variable peptidase activity has been observed in different strains (i.e., iminopeptidase, aminopeptidase $\mathrm{N}$, prolidase, prolyl endopeptidyl peptidase, prolinase, tripeptidase, and dipeptidase). Ten strains, selected to provide the best global peptidase activity needed for completely degrading the immunogenic gluten peptides involved in $\mathrm{CD}$, were pooled and tested for digestion of gliadin peptides. The probiotic mixture was shown to fully hydrolyze immunogenic epitopes, such as the gliadin 33-mer peptide, the peptide spanning residues 57 to 68 of the a9-gliadin ( $\alpha$-gliadin peptide $57-68$ ), A-gliadin peptide 62-75, and $\gamma$-gliadin peptide $62-75$. The selected lactobacilli mixture strongly hydrolyzed the wheat bread gluten (ca. 18,000 ppm) to less than $10 \mathrm{ppm}$ following a treatment lasting $360 \mathrm{~min}$ under simulated GI conditions. The gluten-detoxifying activity was confirmed by culturing duodenal biopsy specimens, obtained from CD patients following a GFD with digested baker's yeast wheat bread in either the absence or the presence of probiotic lactobacilli. The levels of IL-2, IL-10, and IFN- $\gamma$ were overexpressed as a consequence of pepsin-trypsin (PT) wheat bread digestion products that were not treated with the probiotic strains. In contrast, the level of cytokines was similar to the baseline value in duodenal biopsy specimens exposed to PT digestion products from wheat bread, containing the selected probiotic strains (17).

Recently, our group studied the capacity of a probiotic mix (Lactobacillus paracasei, Lactobacillus plantarum, Bifidobacterium animalis subsp. lactis, Bifidobacterium breve Bbr8, Bifidobacterium breve BL10) to hydrolyze gluten peptides following the digestion of gliadin and to modify the pro-inflammatory state as well as the gliadin-induced epithelial modification in the gut (18). The tested lactobacilli and bifidobacteria strains were, indeed, capable of hydrolyzing the fragments of gliadin, produced by digestive proteases, into smaller fragments. In particular, the number of peptides with a molecular weight lower than $3 \mathrm{kDa}$ was significantly higher in the PT-gliadin plus bacteria as compared to PT-gliadin. Moreover, the probiotic strains could degrade the 33-mer immunotoxic peptide in case the compound was the only aminoacid source. Digestion of PT fragments from gliadin with the probiotic strains counteracted the inflammatory state and the disruption of tight junctions induced by PT-gliadin in intestinal epithelial cells.

In vitro and in vivo studies demonstrate that some Bifidobacterium species possess the ability to hydrolyze the toxic gliadin-derived peptides as well as to inhibit the related 
inflammatory response in intestinal epithelial cells. Different Bifidobacterium strains (B. animalis IATA-A2, B. longum IATAES1, B. bifidum IATA-ES) added to a mixture of gliadins during simulated intestinal digestion, produced different peptide patterns with lower molecular mass compared to those noninoculated with bacteria (19). Gliadin-mediated NF-kB activation (responsible for the activation of the pro-inflammatory pathway) and TNF- $\alpha$ levels significantly decreased in intestinal epithelial cells cultures exposed to gliadin digestions inoculated with all bifidobacterial strains mostly in those exposed to digests added with B. longum.

The administration of the same $B$. longum strain has also been shown to decrease jejunal TNF- $\alpha$ levels in an in vivo model of gliadin-induced enteropathy (23). Recently, the effect of Bifidobacterium species, namely B. bifidum, B. animalis, B. breve, and $B$. longum, on the hydrolysis of unmetabolized gluten proteins as well as on the related toxic effect and immune responses elicited by the resulting peptides, has been investigated (20). The different individual species and a mixture of the four Bifidobacterium species resulted in diverse peptide patterns, deriving from the principal wheat protein classes, namely gliadins and glutenins, and presenting from 15 to 40 long amino-acid residues. B. longum showed the highest proteolytic activity toward gluten proteins. In addition, B. longum and the Bifidobacterium consortium possessed a proteolytic activity that acted mostly on gliadin hydrolysis, whereas $B$. animalis favored glutenin digestion. The resulting peptides from the Bifidobacterium cultures had a significantly decreased cytotoxic effect on intestinal cell cultures, especially those produced by B. longum, as compared to gluten-digested peptides from noninoculated samples. The immune response elicited by the gluten-digested peptides from the Bifidobacterium cultures was studied by assaying NF-kB activation and TNF- $\alpha$ and IL-1 $\beta$ expression in cell cultures. As a result, samples containing the peptides from the Bifidobacterium cells displayed a significantly reduced TNF- $\alpha$ expression and NF-kB activation as compared to the positive controls. Furthermore, the decrease in TNF- $\alpha$ levels and NF-kB activation depended on the Bifidobacterium species utilized. In this regard, B. longum and the Bifidobacterium consortium were the most effective in reducing TNF- $\alpha$, and the B. bifidum strain was the least active.

A different approach can also be used to ameliorate gluten intolerance, such as the use of gluten-digesting bacteria to detoxify gluten before the administration to patients. As previously described, the major gluten protein gliadin can be hydrolyzed by lactic acid bacteria under fixed processing conditions $(15,16)$. The use of selected sourdough lactic acid bacteria cultures as starters for fermentation has been suggested as a way to eliminate the risk for gluten contamination. A sourdough containing Lactobacillus plantarum CF1 and Lactobacillus sanfranciscensis LS40 and LS41, chosen for their proteolytic activity, was used for the production of gluten-free bread (24). Gluten was added to the ingredients before fermentation to simulate potential contamination, and as a result, a substantial reduction of gluten concentration (from $400 \mathrm{ppm}$ to below $20 \mathrm{ppm}$ ) was observed. In clinical trials, the administration of fermented foods to $\mathrm{CD}$ subjects was also proved safe $(25,26)$. Baked goods made of wheat flour, rendered gluten-free (gluten to $<10 \mathrm{ppm}$ ) during sourdough fermentation through specific lactobacilli and fungal proteases, were nontoxic to young CD patients (25). Similarly, a 60-day diet of baked goods made from this hydrolyzed form of wheat flour showed no toxicity to CD patients (26).

Our group recently described a protocol for the manufacture of reduced-gluten bread and pasta via fungal proteases as well as selected sourdough lactic acid bacteria. The efficacy of these products (containing $<50 \%$ gluten content) was tested in a randomized, double-blind, crossover-controlled trial of 24 patients with irritable bowel syndrome (IBS) (compared to traditional bread and pasta). We demonstrated that, while consuming bread and pasta with reduced gluten content, IBS patients had significant symptom improvement as measured by the visual analogue scale score $(\mathrm{p}=0.042)(27)$.

At present, in vivo studies fail to provide evidence that probiotic bacteria are able to degrade gluten peptides at a rate capable of guaranteeing that no immunogenic gluten peptides survive in the gut to stimulate CD4 T cells.

\section{PROBIOTICS IN CD PATIENTS}

The theoretical possibility of toxic gliadin peptide digestion led to the hypothesis that probiotics might be useful in patients with CD. Most of the studies have been performed in patients on GFD, and only a few have been carried out in patients on a gluten-containing diet (GCD).

The effect of Bifidobacterium infantis Natren life start strain (NLS-SS) was evaluated in a randomized controlled trial (RCT) on $22 \mathrm{CD}$ patients on GCD (12 g of gluten/day). The authors demonstrate that this specific strain alleviates GI symptoms (gastroesophageal reflux, constipation, and indigestion measured by gastrointestinal symptom rating scale) without any influence on CD serology, gut permeability, growth factors, and cytokines (28). The authors hypothesized that the favorable symptomatic observed effect might be related to an effect on the innate immunity. The mechanisms on innate immunity markers were tested assessing human $\alpha$-defensin 5 (HD5) expression as well as Paneth cells and macrophage counts in duodenal biopsies in three different groups of patients. Thirty-six patients with active $\mathrm{CD}$ were blind randomized to Bifidobacterium infantis (12 patients; group 1) or placebo (24 patients; group 2); $5 \mathrm{CD}$ patients composed the third group after 1 year of GFD. In this study, the authors demonstrated that Bifidobacterium infantis NLS-SS reduces Paneth cell and HD5 expression. On the contrary, GFD induces a more significant reduction of macrophage in duodenum than B. infantis (29).

Håkansson et al. tested the immunomodulatory effect of $L$. plantarum HEAL9 and L. paracasei 8700:2 in pediatric patients with CD autoimmunity before diagnosis and GFD. They studied 78 children with CD autoimmunity for 6 months (40 received the two lactobacilli and 38 placebo). The authors found significant changes in the peripheral immune response implicated in T-cell regulation only in children that received placebo. The results show that $L$. paracasei and $L$. plantarum may play a regulatory 
role on the peripheral immune response in $\mathrm{CD}$. Moreover, the authors found a more significant reduction of the levels of IgAtTG in the probiotic ( $p=0.013$ ) compared to placebo (30).

The effect of the administration of a mixture of two Bifidobacterium breve strains (B632 and BR03) on microbiota modulation in CD children on a GFD was evaluated by Quagliarello et al. The study population consisted of $40 \mathrm{CD}$ children (randomly allocated into two groups: 20 in the probiotic group and 20 in the placebo group) and 16 healthy children as controls. The authors revealed an imbalance of the intestinal microbial composition of CD patients mainly characterized by a reduction of the Firmicutes/Bacteroidetes ratio of Actinobacteria and Euryarchaeota compared to the controls. The supplementation was able to induce an increase of Actinobacteria as well as a restoration of the Firmicutes/Bacteroidetes ratio (31).

Klemenak et al. investigate the same mixture of Bifidobacterium breve strains (BR03 and B632). The authors randomized $49 \mathrm{CD}$ children (on GFD) in two groups (Bifidobacterium breve strains BR03 and B632 vs. placebo) and demonstrate a reduction of TNF- $\alpha$ levels in the probiotic group after receiving $B$. breve for 3 months. However, the effect of the supplementation is not durable: TNF- $\alpha$ levels increased again 3 months after completion of the intervention. The authors did not reveal any difference in IL-10 levels between the two groups (32).

Recently an RCT on 40 children with CD and 16 healthy controls was performed to evaluate the effect of a mixture of two Bifidobacterium breve strains (DSM 16604 and DSM 24706). The result showed TNF- $\alpha$ level reduction and reestablishment of the Firmicutes/Bacteroidetes ratio after 3 months of probiotic administration (33).

Olivares et al. demonstrate, in patients on GFD, that Bifidobacterium longum CECT 7347 led to a reduction in activated T-lymphocytes and TNF- $\alpha$ levels as compared to placebo. Moreover, the increase in height percentile was significantly greater in patients who assumed probiotic compared to those who assumed placebo $(p<0 \cdot 048)$ although weight percentile was similar in the two groups. The probiotic treatment also induced a significant decrease in the Bacteroides fragilis and content of sIgA in stools. No differences in GI symptoms were observed between probiotic and placebo groups (34). Our group performed an RCT on 109 CD patients with IBS symptoms despite GFD; we assessed the role of a probiotics mixture made up offive strains of lactic acid bacteria and Bifidobacteria [Lactobacillus casei LMG 101/37 P17504 ( $5 \times 10^{9} \mathrm{CFU} /$ sachet), Lactobacillus plantarum CECT 4528 $\left(5 \times 10^{9} \mathrm{CFU} / \mathrm{sach}\right.$ t), Bifidobacterium animalis subsp. lactis Bi1 LMG P-17502 (10x10 ${ }^{9} \mathrm{CFU} /$ sachet), Bifidobacterium breve

\section{REFERENCES}

1. Husby S, Koletzko S, Korponay-Szabó I, Kurppa K, Mearin ML, RibesKoninckx C, et al. European Society Paediatric Gastroenterology, Hepatology and Nutrition Guidelines for Diagnosing Coeliac Disease 2020. J Pediatr Gastroenterol Nutr (2020) 70:141-56. doi: 10.1097/MPG.0000000000002497

2. Barone M, Della Valle N, Rosania R, Facciorusso A, Trotta A, Cantatore FP, et al. A comparison of the nutritional status between adult celiac patients on a long-term, strictly gluten-free diet and healthy subjects. Eur J Clin Nutr (2016) 70:23-7. doi: 10.1038/ejen.2015.114
Bbr8 LMG P-17501 (10x109 CFU/sachet), Bifidobacterium breve Bl10 LMG P-17500 (10x10 ${ }^{9}$ CFU/sachet)] administered for 6 weeks followed by a follow-up period of 6 more weeks. We demonstrate that the probiotic mixture was able to improve the severity of IBS symptoms. In detail, we showed a higher percentage of treatment success (defined as a decrease of at least $50 \%$ of IBS severity score) at both intention-to-treat $(14.8 \%$ vs. $3.6 \%$; $p<0.04)$ and per-protocol analysis $(15.3 \%$ vs. $3.8 \%$; $p<0.04)$ after 6 weeks of treatment. Moreover, the probiotic mixture exerted a positive microbiota modulation with a durable increase of bifidobacteria persistent 6 weeks after the end of the treatment (35).

Finally, Harnett et al. studied $45 \mathrm{CD}$ patients complaining of GI symptoms despite strict adherence to GFD for 12 months. Participants were randomized to $5 \mathrm{~g}$ of VSL\#3 probiotic formulation or placebo for 3 months. The results revealed no statistically significant changes in the fecal microbiota between the groups (36).

Overall, no study has demonstrated that supplementation of probiotics to untreated CD subjects leads to normalization of the gut histology or prevents that changes occur in the mucosal architecture on long term after oral gluten challenge; therefore, all the studies are to be considered preliminary and without an application in the clinical practice.

\section{CONCLUSION}

Several shreds of evidence demonstrate that probiotics are an excellent resource of endopeptidases for digestion and reduction of gluten toxicity; however, we are far from a possible application in the clinical practice. We firmly believe that, at present, GFD is the only therapeutic option for CD patients and that it must be rigorous and permanent. A future challenge is the possible application of the biochemical machinery of the bacterial endopeptidases to digest the gluten toxic epitopes to be used to produce a wheat gluten-free flour maintaining the nutritional value of this prohibited cereal. The idea that the use of probiotics can allow even minimal transgressions to the diet must be discouraged because, at the moment, there is no data to support this hypothesis.

\section{AUTHOR CONTRIBUTIONS}

All authors have participated in drafting and revising the review, and they have seen and approved the final version. All authors take full responsibility for the manuscript. All authors contributed to the article and approved the submitted version.
3. Lynch SV, Pedersen O. The Human Intestinal Microbiome in Health and Disease. N Engl J Med (2016) 375(24):2369-79. doi: 10.1056/NEJMra1600266

4. Galipeau HJ, McCarville JL, Huebener S, Litwin O, Meisel M, Jabri B, et al. Intestinal microbiota modulates gluten-induced immunopathology in humanized mice. Am J Pathol (2015) 185:2969-82. doi: 10.1016/ j.ajpath.2015.07.018

5. Sanz Y. Microbiome and Gluten. Ann Nutr Metab (2015) 67(2):28-41. doi: 10.1159/000440991

6. Hill C, Guarner F, Reid G, Gibson GR, Merenstein DJ, Pot B, et al. The International Scientific Association for Probiotics and Prebiotics consensus 
statement on the scope and appropriate use of the term probiotic. Nat Rev Gastroenterol Hepatol (2014) 11:506-14. doi: 10.1038/nrgastro.2014.66

7. Pecora F, Persico F, Gismondi P, Fornaroli F, Iuliano S, de'Angelis GL, et al. Gut Microbiota in Celiac Disease: Is There Any Role for Probiotics? Front Immunol (2020) 11:957:957. doi: 10.3389/fimmu.2020.00957

8. Shan L, Molberg Ø, Parrot I, Hausch F, Filiz F, Gray GM, et al. Structural basis for gluten intolerance in celiac sprue. Science (2002) 297:2275-9. doi: 10.1126/ science. 1074129

9. Tye-Din JA, Stewart JA, Dromey JA, Beissbarth T, van Heel DA, Tatham A, et al. Comprehensive, Quantitative Mapping of T Cell Epitopes in Gluten in Celiac Disease. Sci Transl Med (2010) 2:41ra51-1. doi: 10.1126/ scitranslmed.3001012

10. Duar RM, Clark KJ, Patil PB, Hernández C, Brüning S, Burkey TE, et al. Identification and characterization of intestinal lactobacilli strains capable of degrading immunotoxic peptides present in gluten. J Appl Microbiol (2015) 118:515-27. doi: 10.1111/jam.1268

11. Caminero A, Herrán AR, Nistal E, Pérez-Andrés J, Vaquero L, Vivas S, et al. Diversity of the cultivable human gut microbiome involved in gluten metabolism: isolation of microorganisms with potential interest for coeliac disease. FEMS Microbiol Ecol (2014) 88:309-19. doi: 10.1111/1574-6941.12295

12. Herran AR, Pérez-Andrés J, Caminero A, Nistal E, Vivas S, Ruiz de Morales JM, et al. Gluten-degrading bacteria are present in the human small intestine of healthy volunteers and celiac patients. Res Microbiol (2017) 168:673-84. doi: 10.1016/j.resmic.2017.04.008

13. Sánchez E, Laparra JM, Sanz Y. Discerning the role of Bacteroides fragilis in celiac disease pathogenesis. Appl Environ Microbiol (2012) 78:6507-15. doi: 10.1128/AEM.00563-12

14. Caminero A, Galipeau HJ, McCarville JL, Johnston CW, Bernier SP, Russell AK, et al. Duodenal bacteria from patients with celiac disease and healthy subjects distinctly affect gluten breakdown and immunogenicity. Gastroenterology (2016) 151(4):670-83. doi: 10.1053/j.gastro.2016.06.041

15. Di Cagno R, De Angelis M, Auricchio S, Greco L, Clarke C, De Vincenzi M, et al. Sourdough bread made from wheat and nontoxic flours and started with selected lactobacilli is tolerated in celiac sprue patients. Appl Environ Microbiol (2004) 70:1088-96. doi: 10.1128/aem.70.2.1088-1096.2004

16. De Angelis M, Rizzello CG, Fasano A, Clemente MG, De Simone C, Silano M, et al. VSL3 probiotic preparation has the capacity to hydrolyze gliadin polypeptides responsible for celiac sprue. Biochim Biophys Acta (2006) 1762:80-93. doi: 10.1016/j.bbadis.2005.09.008

17. Francavilla R, De Angelis M, Rizzello CG, Cavallo N, Dal Bello F, Gobbetti M. Selected Probiotic Lactobacilli Have the Capacity To Hydrolyze Gluten Peptides during Simulated Gastrointestinal Digestion. Appl Environ Microbiol (2017) 83:e00376-17. doi: 10.1128/AEM.00376-1

18. Giorgi A, Cerrone R, Capobianco D, Filardo S, Mancini P, Zanni F, et al. A Probiotic Preparation Hydrolyzes Gliadin and Protects Intestinal Cells from the Toxicity of Pro-Inflammatory Peptides. Nutrients (2020) 12:495. doi: 10.3390/nu12020495

19. Laparra JM, Sanz Y. Bifidobacteria inhibit the inflammatory response induced by gliadins in intestinal epithelial cells via modifications of toxic peptide generation during digestion. J Cell Biochem (2010) 109:801-7. doi: 10.1002/ jcb.22459

20. Castilho de Almeida NE, Esteves FG, Dos Santos-Pinto JRA, Peres de Paula C, Ferreira da Cunha A, Iran Malavazi I, et al. Digestion of Intact Gluten Proteins by Bifidobacterium Species: Reduction of Cytotoxicity and Proinflammatory Responses. J Agric Food Chem (2020) 68:4485-92. doi: 10.1021/ acs.jafc.0c01421

21. Christensen JE, Dudley EG, Pederson JA, Steele JL. Peptidases and amino acid catabolism in lactic acid bacteria. Antonie Van Leeuwenhoek (1999) 76:21746. doi: 10.1007/978-94-017-2027-4_11

22. De Angelis M, Cassone A, Rizzello CG, Gagliardi F, Minervini F, Calasso M, et al. Mechanism of degradation of immunogenic gluten epitopes from Triticum turgidum L. var. durum by sourdough lactobacilli and fungal proteases. Appl Environ Microbiol (2010) 76:508-518. doi: 10.1128/AEM.01630-09

23. Laparra JM, Olivares M, Gallina O, Sanz Y. Bifidobacterium longum CECT 7347 modulates immune responses in a gliadin-induced enteropathy animal model. PloS One (2012) 7:e30744. doi: 10.1371/journal.pone.0030744

24. Di Cagno R, Rizzello CG, De Angelis M, Cassone A, Giuliani G, Benedusi A, et al. Use of selected sourdough strains of Lactobacillus for removing gluten and enhancing the nutritional properties of gluten-free bread. J Food Prot (2008) 71(7):1491-95. doi: 10.4315/0362-028x-71.7.1491

25. Di Cagno R, Barbato M, Di Camillo C, Rizzello CG, De Angelis M, Giuliani G, et al. Gluten-free sourdough wheat baked goods appear safe for young celiac patients: a pilot study. J Pediatr Gastroenterol Nutr (2010) 51:777-83. doi: 10.1097/MPG.0b013e3181f22ba4

26. Greco L, Gobbetti M, Auricchio R, Di Mase R, Landolfo F, Paparo F, et al. Safety for patients with celiac disease of baked goods made of wheat flour hydrolyzed during food processing. Clin Gastroenterol Hepatol (2011) 9 (1):24-9. doi: 10.1016/j.cgh.2010.09.025

27. Calasso M, Francavilla R, Cristofori F, De Angelis M, Gobbetti M. New Protocol for Production of Reduced-Gluten Wheat Bread and Pasta and Clinical Effect in Patients with Irritable Bowel Syndrome: A randomised, Double-Blind, Cross-Over Study. Nutrients (2018) 10:1873. doi: 10.3390/ nu10121873

28. Smecuol E, Hwang HJ, Sugai E, Corso L, Cherñavsky AC, Bellavite FP, et al. Exploratory, randomized, double-blind, placebo-controlled study on the effects of Bifidobacterium infantis Natren life start strain super strain in active celiac disease. J Clin Gastroenterol (2013) 47:139-47. doi: 10.1097/ MCG.0b013e31827759ac

29. Pinto-Sánchez MI, Smecuol EC, Temprano MP, Sugai E, González A, Moreno ML, et al. Bifidobacterium infantis NLS Super Strain Reduces the Expression of $\alpha$-Defensin-5, a Marker of Innate Immunity, in the Mucosa of Active Celiac Disease Patients. J Clin Gastroenterol (2017) 51:814-7. doi: 10.1097/ MCG.0000000000000687

30. Håkansson Å, Andrén Aronsson C, Brundin C, Oscarsson E, Molin G, Agardh D. Effects of Lactobacillus plantarum and Lactobacillus paracasei on the Peripheral Immune Response in Children with Celiac Disease Autoimmunity: A Randomized, Double-Blind, Placebo-Controlled Clinical Trial. Nutrients (2019) 11:1925. doi: 10.3390/nu11081925

31. Quagliariello A, Aloisio I, Bozzi Cionci N, Luiselli D, D’Auria G, MartinezPriego L, et al. Effect of Bifidobacterium breve on the Intestinal Microbiota of Coeliac Children on a Gluten Free Diet: A Pilot Study. Nutrients (2016) 8:660. doi: $10.3390 /$ nu 8100660

32. Klemenak M, Dolinšek J, Langerholc T, Di Gioia D. Administration of Bifidobacterium breve Decreases the Production of TNF- $\alpha$ in Children with Celiac Disease. Dig Dis Sci (2015) 60:3386-92. doi: 10.1007/s10620-015-3769-7

33. Primec M, Klemenak M, Di Gioia D, Aloisio I, Bozzi Cionci N, Quagliariello A, et al. Clinical intervention using Bifidobacterium strains in celiac disease children reveals novel microbial modulators of TNF- $\alpha$ and short-chain fatty acids. Clin Nutr (2019) 38:1373-81. doi: 10.1016/j.clnu.2018.06.931

34. Olivares M, Castillejo G, Varea V, Sanz Y. Double-blind, randomised, placebo-controlled intervention trial to evaluate the effects of Bifidobacterium longum CECT 7347 in children with newly diagnosed coeliac disease. Br J Nutr (2014) 112:30-40. doi: 10.1017/S0007114514000609

35. Francavilla R, Piccolo M, Francavilla A, Polimeno L, Semeraro F, Cristofori F, et al. Clinical and Microbiological Effect of a Multispecies Probiotic Supplementation in Celiac Patients with Persistent IBS-type Symptoms: A Randomized, Double-Blind, Placebo-controlled, Multicenter Trial. J Clin Gastroenterol (2018) 53(3):e117-25. doi: 10.1097/MCG.0000000000001023

36. Harnett J, Myers SP, Rolfe M. Probiotics and the microbiome in celiac disease: A randomised controlled trial. Evid Based Complement Alternat Med (2016) 2016:9048574. doi: 10.1155/2016/9048574

Conflict of Interest: RF is the inventor of the patent N 0001425900, released on November 17, 2016 (Italy).

The remaining authors declare that the research was conducted in the absence of any commercial or financial relationships that could be construed as a potential conflict of interest.

Copyright (c) 2020 Cristofori, Francavilla, Capobianco, Dargenio, Filardo and Mastromarino. This is an open-access article distributed under the terms of the Creative Commons Attribution License (CC BY). The use, distribution or reproduction in other forums is permitted, provided the original author(s) and the copyright owner(s) are credited and that the original publication in this journal is cited, in accordance with accepted academic practice. No use, distribution or reproduction is permitted which does not comply with these terms. 